\title{
CENTRAL INTERPOLATION SETS FOR COMPACT GROUPS AND HYPERGROUPS
}

\author{
DAVID GROW \\ Department of Mathematics and Statistics, University of Missouri-Rolla, Rolla, MO 65409, USA \\ e-mail: grow@mst.edu \\ and KATHRYN E. HARE \\ Department of Pure Mathematics, University of Waterloo, Waterloo, Ontario N2L 3G1, Canada \\ e-mail:kehare@uwaterloo.ca
}

(Received 21 June 2007; accepted 17 April 2009)

\begin{abstract}
We prove that every infinite subset of the dual of a compact, connected group contains an infinite, central, weighted $I_{0}$ set. This yields a new proof of the fact that the duals of such groups admit infinite central $p$-Sidon sets for each $p>1$. We also establish the existence of infinite, weighted $I_{0}$ sets in the duals of many compact, abelian hypergroups.
\end{abstract}

2000 Mathematics Subject Classification. Primary 43A46; secondary 43A62, $43 \mathrm{~A} 30$.

1. Introduction. A subset $E$ of the dual of a compact group $G$ is called a Sidon set (resp. $I_{0}$ set $^{1}$ ) if every bounded $E$-function can be interpolated by the Fourier transform of a (resp. discrete) measure on $G$. Sidon sets in duals of compact, abelian groups have been extensively studied and found to be very useful. Although there are examples of Sidon sets that are not $I_{0}$, both classes are plentiful. Indeed, every infinite subset of $\widehat{G}$ contains an infinite $I_{0}$ set; for recent proofs see $[4,7,10]$. Other recent research has emphasised the study of particular classes of examples of $I_{0}$ sets in which further properties are imposed on the interpolating measure or classes with more structure such as Hadamard and $\varepsilon$-Kronecker sets (c.f. [6, 9, 17],

In contrast, there are compact, non-abelian groups whose duals admit no infinite (central) Sidon sets $[\mathbf{1}, \mathbf{2 0}]$. Sidon sets in compact, connected, non-abelian groups have been essentially characterised, and this characterisation has been used to prove that every infinite Sidon set contains an infinite $I_{0}$ set and that the interpolating measure can be taken to be real or supported on a small set, in addition to discrete $[\mathbf{8}, \mathbf{1 4}]$.

The non-existence of infinite Sidon sets in many non-abelian groups is a consequence of the unboundedness of the degrees of the representations and motivated the introduction of weighted Sidon-type sets in [15], where the effect of the degree is dampened.

In this paper we extend this notion to $I_{0}$ sets, and we prove that every infinite subset of the dual of any compact connected group contains weighted central $I_{0}$ sets. Our approach is quite different from the earlier work on the existence of central

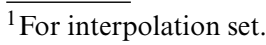


Sidon-type sets because we construct the interpolating measure, rather than using the dual method of bounding norms of suitable polynomials as in $[\mathbf{3}, \mathbf{1 2}, \mathbf{1 8}]$, for example.

We also study the more general problem of weighted $I_{0}$ sets in the duals of compact, abelian hypergroups, improving upon the results in [13]

2. Weighted central $I_{0}$ sets on groups. Let $G$ be a compact group, and denote by $\widehat{G}$ its dual object, a maximal set of irreducible, inequivalent representations of $G ; M(G)$ will denote the space of finite, regular, Borel measures on $G$. We let $\operatorname{deg} \sigma$ denote the degree of the representation $\sigma \in \widehat{G}$ and $H_{\sigma}$ the complex Hilbert space of dimension $\operatorname{deg} \sigma$ on which it acts. The norm of a matrix $A \in B\left(H_{\sigma}\right)$ will be the usual operator norm and will be denoted $\|A\|_{\infty}$.

Suppose $E \subseteq \widehat{G}$. Given $a \in \mathbb{R}$ and $A=\left(A_{\sigma}\right)_{\sigma \in E}$ with $A_{\sigma} \in B\left(H_{\sigma}\right)$, we let

$$
\|A\|_{a, \infty}=\sup \left\{(\operatorname{deg} \sigma)^{a}\left\|A_{\sigma}\right\|_{\infty}: \sigma \in E\right\}
$$

and we denote by $l_{a, \infty}(E)$ the weighted $l_{\infty}$ space,

$$
l_{a, \infty}(E)=\left\{A=\left(A_{\sigma}\right)_{\sigma \in E}:\|A\|_{a, \infty}<\infty\right\} .
$$

When $a=0$ we have the usual $l_{\infty}$ space. By $l_{a, \infty}^{z}(E)$ we will mean the subset of $l_{a, \infty}(E)$ with $A=\left(c_{\sigma} I_{\operatorname{deg} \sigma}\right)_{\sigma \in E}$.

A subset $E \subseteq \widehat{G}$ is called Sidon $\left(I_{0}\right)$ if whenever $A \in l_{\infty}(E)$, there is a (discrete) measure $\mu$ on $G$ such that $\widehat{\mu}(\sigma)=\phi(\sigma)$ for all $\sigma \in E$. Finite sets are always Sidon $/ I_{0}$; hence the interest is in infinite sets. When $G$ is abelian these sets are abundant. Examples include lacunary sets in $\mathbb{Z}$ and linearly independent sets. Every infinite subset in the dual of an infinite abelian group contains an infinite Sidon set, and any finite subset of a Sidon set contains an $I_{0}$ set of proportionate size [21]. An open problem is to determine if every Sidon set is a finite union of $I_{0}$ sets.

Motivated by the fact that there are no infinite Sidon sets in the dual of any compact, simple, connected Lie group, the weaker notion of (central)( $a, p)$-Sidon sets was introduced in [15]. Our interest is in the case $p=1$, and we extend the definition to $(a, 1)-I_{0}$ sets.

Recall that a measure is called central if it commutes with all other measures on the group under convolution. Central measures are characterised by the property that their Fourier transforms are scalar multiples of identity matrices.

Definition 2.1. (i) A subset $E \subseteq \widehat{G}$ is called an (a,1)-Sidon (resp. $\left.(a, 1)-I_{0}\right)$ set if whenever $\phi \in l_{1-a, \infty}(E)$, there is a (resp. discrete) measure $\mu \in M(G)$ such that $\widehat{\mu}(\sigma)=\phi(\sigma)$ for all $\sigma \in E$.

(ii) If each $\phi \in l_{1-a, \infty}^{z}(E)$ can be interpolated on $E$ by the Fourier transform of a central measure $\mu \in M(G)$, then $E$ is known as a central $(a, 1)$-Sidon set.

A $(1,1)$-Sidon (or $(1,1)-I_{0}$ ) set is Sidon (resp. $I_{0}$ ), and since $l_{1-a, \infty} \subseteq l_{1-b, \infty}$ if $a \leq b$, it is formally easier to be an $(a, 1)$-Sidon $\left(I_{0}\right)$ set as $a$ decreases. Of course, if $G$ is abelian, there is no distinction between the classes as $a$ varies, since the degree of any $\sigma \in \widehat{G}$ is one. It is known that Sidon sets are central Sidon, but the converse is false [18].

Given the relationship between Sidon sets and $I_{0}$ sets, it would be natural to define a central $(a, 1)-I_{0}$ set as one for which the interpolating measure in the definition of a central $(a, 1)$-Sidon set could be chosen to be both central and discrete. However, if this were taken as the definition, not even all finite sets would be central $I_{0}$. This is 
because in connected groups any discrete central measure is supported on the centre of the group [20], and there are groups with finite centres.

Instead, we will replace central discrete measures by orbital measures: the orbital measure $\mu_{x}$, for $x \in G$, is the probability measure supported on the conjugacy class containing $x$ and defined by

$$
\int_{G} f d \mu_{x}=\int_{G} f\left(g x g^{-1}\right) d m_{G}(g)
$$

for all continuous functions $f$ on $G$. (Here $m_{G}$ denotes Haar measure on $G$.) Orbital measures are always central, and their Fourier transforms are given by

$$
\widehat{\mu_{x}}(\sigma)=(\operatorname{Tr} \sigma(x) / \operatorname{deg} \sigma) I_{\operatorname{deg} \sigma} .
$$

We put forth the following definition.

Definition 2.2. A subset $E \subseteq \widehat{G}$ is called a central $(a, 1)-I_{0}$ set if whenever $\phi \in$ $l_{1-a, \infty}^{z}(E)$, there is a sum of orbital measures, $\mu=\sum b_{k} \mu_{x_{k}} \in M(G)$, such that $\widehat{\mu}(\sigma)=$ $\phi(\sigma)$ for all $\sigma \in E$.

Proposition 2.1. [11, Proposition 4.1] Any $(a, 1)-I_{0}$ set in $\widehat{G}$ is central $(a, 1)-I_{0}$.

COROLlary 2.2. Finite sets are central $(a, 1)-I_{0}$ for all $a$.

As with central weighted Sidon sets there are a number of properties equivalent to the definition of central weighted $I_{0}$.

Proposition 2.3. Let $G$ be a compact group. The following are equivalent for $E \subset \widehat{G}$ :

(1) The set $E$ is a central $(1-a, 1)-I_{0}$ set.

(2) There is a constant $C$ such that whenever $\phi \in l_{a, \infty}^{z}(E)$ there is a measure $\mu=$ $\sum b_{k} \mu_{x_{k}}$ such that $\widehat{\mu}(\sigma)=\phi(\sigma)$ for all $\sigma \in E$ and $\|\mu\| \leq C\|\phi\|_{a, \infty}$.

(3) For every $0<\varepsilon<1$ (equivalently, there exists $0<\varepsilon<1$ ) for which there is a constant $C$ so that whenever $\phi \in l_{a, \infty}^{z}(E)$ there is a measure $\mu=\sum b_{k} \mu_{x_{k}}$ such that $\left\|\left.\widehat{\mu}\right|_{E}-\phi\right\|_{a, \infty} \leq \varepsilon\|\phi\|_{a, \infty}$ and $\|\mu\| \leq C\|\phi\|_{a, \infty}$.

(4) For every $0<\varepsilon<1$ (equivalently, there exists $0<\varepsilon<1$ ) for which there is a constant $C$ so that for each choice of $\left\{r_{\sigma}\right\}_{\sigma \in E}, r_{\sigma}= \pm 1$, there is a measure $\mu=\sum b_{k} \mu_{x_{k}}$ such that $\|\mu\| \leq C$ and

$$
\sup \left\{(\operatorname{deg} \sigma)^{a}\left\|\widehat{\mu}(\sigma)-\frac{r_{\sigma} I_{\operatorname{deg} \sigma}}{(\operatorname{deg} \sigma)^{a}}\right\|_{\infty}: \sigma \in E\right\} \leq \varepsilon .
$$

Proofs of similar results can be found in [11] and [21], for example.

It follows easily from (4) that the independent sets of [18] are examples of central $I_{0}$ sets. Other examples of weighted $I_{0}$ sets can be found in [11] in which the problem of approximating signs by characters is studied in the non-abelian setting. The main results of that paper, as applied to the study of $I_{0}$ sets, are summarised below.

Proposition 2.4. [11]

(1) If $G$ is any infinite, compact, connected group, then $\widehat{G}$ contains an infinite central $(0,1)-I_{0}$ set.

(2) If $G$ is any compact, simple, simply connected, connected Lie group, other than $S U(2)$ or $S U(3)$, then $\widehat{G}$ contains an infinite central $(a, 1)-I_{0}$ set for some $a>0$. 
3. Existence of weighted central $I_{0}$ sets in compact, connected groups. In this section we will prove that every infinite subset of the dual of a compact, connected group contains infinite weighted central $I_{0}$ sets. We prove this first for infinite products of simple Lie groups, and then we appeal to the structure theorem for compact, connected groups.

THEOREM 3.1. Let $G=\prod G_{i}$ be a product of compact, simple, connected, simply connected Lie groups and let $a<1$. Then any infinite subset of $\widehat{G}$ of unbounded degree contains an infinite, central $(a, 1)-I_{0}$ set.

Proof. Let $\left\{\sigma_{j}\right\} \subseteq \widehat{G}$ be a set of unbounded degree. We will select a suitable infinite subset of $\left\{\sigma_{j}\right\}$ through an inductive process, and during this process we will also identify points $x_{j} \in G$ which will be used, later in the proof, to build the interpolating measures.

Without loss of generality we can assume $\delta=1-a<1 / 2$ and $\sigma_{j} \neq 1$ for any $j$. Let $\pi_{i}: G_{i} \rightarrow G$ be the natural embedding map. Note that for each $j, \sigma_{j} \circ \pi_{i}$ is trivial for all but finitely many $i$; let $I_{1}=\left\{i: \sigma_{1} \circ \pi_{i} \neq 1\right\}$. We can view $\sigma_{1}$ as a representation on $H_{1} \equiv \prod_{i \in I_{1}} G_{i}$ in the natural way.

The trace of a representation at elements of the torus can be calculated by the Weyl character formula (see [24, Theorem 4.14.4]). The formula is a quotient, $P / Q$, where $Q$ is independent of the particular representation and $|P|$ is at most the cardinality of the Weyl group of the semi-simple Lie group $H_{1}$. The elements at which $Q$ is non-zero are called regular, and these are dense in the torus.

Choose a regular torus element, $y_{1}=\left(x_{1 j}\right)_{j \in I_{1}} \in H_{1}$, with the property that

$$
\left|\operatorname{Tr} \sigma_{1}\left(y_{1}\right)-\operatorname{deg} \sigma_{1}\right| \leq 1 / 6 .
$$

As $y_{1}$ is regular there is a constant $A_{1}$, depending on $H_{1}$ and $y_{1}$, such that $\left|\operatorname{Tr} \sigma\left(y_{1}\right)\right| \leq A_{1}$ for all $\sigma \in \widehat{H}_{1}$.

Fix $q>6^{1 / \delta}$, set $n_{1}=1$ and choose $n_{2}>n_{1}$ such that

$$
\operatorname{deg} \sigma_{n_{2}} \geq q \max \left(\operatorname{deg} \sigma_{n_{1}}, A_{1}^{1 /(1-\delta)}\right) .
$$

We have $\sigma_{n_{2}}=\alpha_{2}^{(1)} \times \alpha_{2}^{(2)}$ for some $\alpha_{2}^{(1)} \in \widehat{H_{1}}$ and $\alpha_{2}^{(2)} \in \widehat{H_{2}} \equiv \widehat{\prod_{i \in I_{2}} G_{i}}$, with $I_{1}, I_{2}$ disjoint sets of indices. If $\alpha_{2}^{(2)}=1$, then clearly $\operatorname{Tr} \alpha_{2}^{(2)}(x)=1$ for all $x \in H_{2}$. Otherwise, $\operatorname{Tr} \alpha_{2}^{(2)}$ has a root in $H_{2}$ [5], and so by continuity we can find a regular element $\left(x_{1 j}\right)_{j \in I_{2}} \in H_{2}$ such that

$$
\left|\operatorname{Tr}_{2}^{(2)}\left(\left(x_{1 j}\right)_{j \in I_{2}}\right)\right| \leq 1
$$

Let $J_{2}=I_{1} \bigcup I_{2}$, and choose a regular element $y_{2}=\left(x_{2 j}\right)_{j \in J_{2}}$, with $x_{2 j} \neq x_{1 j}$ for some $j \in J_{2}$ and satisfying

$$
\left|\operatorname{Tr}_{n_{2}}\left(y_{2}\right)-\operatorname{deg} \sigma_{n_{2}}\right| \leq 1 / 6
$$

Then find $A_{2}$ such that for $i=1,2$ and all $\lambda \in{\widehat{H_{1} \times H_{2}}}_{2}$,

$$
\left|\operatorname{Tr} \lambda\left(\left(x_{i j}\right)_{j \in I_{1} \cup I_{2}}\right)\right| \leq A_{2} \text {. }
$$

We have now chosen two of the representations which will be in the infinite central $(a, 1)-I_{0}$ subset, namely $\sigma_{n_{1}}$ and $\sigma_{n_{2}}$, and have determined the $J_{2}$ coordinates of the 
points $x_{1}$ and $x_{2}$, namely $x_{1 j}, x_{2 j}$ for $j \in J_{2}$. We continue the construction by proceeding inductively.

Assume that for $k=1, \ldots, N$ we have chosen representations

$$
\sigma_{n_{k}}=\alpha_{k}^{(1)} \times \cdots \times \alpha_{k}^{(k)}, k=1, \ldots, N, \text { where } \alpha_{k}^{(j)} \in \widehat{H}_{j} \equiv \widehat{\prod_{i \in I_{j}} G_{i}},
$$

constants $A_{k}$ and distinct, regular elements $\left(x_{i j}\right) \in \prod_{i \in J_{k}} G_{i}$ for $i \leq k$ and $J_{k}=\bigcup_{l=1}^{k} I_{l}$ with the following properties:

(i) for $y_{k}=\left(x_{k j}\right)_{j \in J_{k}}$ we have $\left|\operatorname{Tr} \sigma_{n_{k}}\left(y_{k}\right)-\operatorname{deg} \sigma_{n_{k}}\right| \leq 1 / 6$;

(ii) $\left|\operatorname{Tr} \alpha_{k}^{(k)}\left(\left(x_{i j}\right)_{j \in I_{k}}\right)\right| \leq 1$ for all $i \leq k-1$;

(iii) $\left|\operatorname{Tr} \lambda\left(\left(x_{i j}\right)_{j \in J_{k}}\right)\right| \leq A_{k}$ for all $\lambda \in \widehat{\prod_{i=1}^{k} H_{i}}$ and $i=1, \ldots, k$; and

(iv) $\operatorname{deg} \sigma_{n_{k}} \geq q \max \left(\operatorname{deg} \sigma_{n_{k-1}}, A_{k-1}^{1 /(1-\delta)}\right.$ ) (for $k \geq 2$ ).

Next, select $n_{N+1}>n_{N}$ so large that

$$
\operatorname{deg} \sigma_{n_{N+1}} \geq q \max \left(\operatorname{deg} \sigma_{n_{N}}, A_{N}^{1 /(1-\delta)}\right)
$$

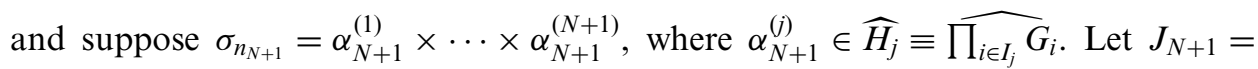
$\bigcup_{l=1}^{N+1} I_{l}$. Choose a regular element $\left(x_{1 j}\right)_{j \in I_{N+1}}$, such that

$$
\left|\operatorname{Tr}_{N+1}^{(N+1)}\left(\left(x_{1 j}\right)_{j \in I_{N+1}}\right)\right| \leq 1
$$

and set $x_{i j}=x_{1 j}$ for $j \in I_{N+1}$ and $i \leq N$. (So now the $I_{N+1}$ coordinates of $x_{1}, \ldots, x_{N}$ have been determined.) Select a regular element $y_{N+1} \in \prod_{j=1}^{N+1} H_{j}, y_{N+1} \neq\left(x_{i j}\right)_{J_{N+1}}$ for $i \leq N$, satisfying

$$
\left|\operatorname{Tr} \sigma_{n_{N+1}}\left(y_{N+1}\right)-\operatorname{deg} \sigma_{n_{N+1}}\right| \leq 1 / 6 \text {. }
$$

To complete the induction step we specify the $J_{N+1}$ coordinates of $x_{N+1}$ by setting them equal to $y_{N+1}$, and we choose $A_{N+1}$ so that for all $\lambda \in \widehat{\prod_{j=1}^{N+1} H_{j}}$ and $i=1, \ldots, N+1$ we have $\left|\operatorname{Tr} \lambda\left(\left(x_{i j}\right)_{j \in J_{N+1}}\right)\right| \leq A_{N+1}$.

We will now verify that $\left\{\sigma_{n_{j}}\right\}$ is a central $(a, 1)-I_{0}$ set. So let $\left\{r_{i}\right\}$ be a choice of signs and put

$$
\mu=\sum_{i} r_{i} \frac{\mu_{x_{i}}}{\left(\operatorname{deg} \sigma_{n_{i}}\right)^{\delta}}
$$

where $x_{i}=\left(x_{i j}\right)_{j}$ were defined through the inductive construction. (Put $x_{i j}=e$ for any unspecified coordinates.) This is a finite measure since the degrees grow exponentially. Of course,

$$
\widehat{\mu}\left(\sigma_{n_{k}}\right)=\frac{1}{\operatorname{deg} \sigma_{n_{k}}}\left(\sum_{i} r_{i} \frac{\operatorname{Tr} \sigma_{n_{k}}\left(x_{i}\right)}{\operatorname{deg} \sigma_{n_{i}}^{\delta}}\right) I_{\operatorname{deg} \sigma_{n_{k}}} .
$$


Recall that $\sigma_{n_{k}}=\alpha_{k}^{(1)} \times \cdots \times \alpha_{k}^{(k-1)} \times \alpha_{k}^{(k)}$, where $\alpha_{k}^{(j)} \in \widehat{H}_{j}$. The choice of $A_{k-1}$ (property (iii)) ensures that if $i \leq k-1$, then

$$
\left|\operatorname{Tr} \alpha_{k}^{(1)} \times \cdots \times \alpha_{k}^{(k-1)}\left(\left(x_{i j}\right)_{j \in J_{k}}\right)\right| \leq A_{k-1} .
$$

Property (ii) gives that $\left|\operatorname{Tr} \alpha_{k}^{(k)}\left(\left(x_{i j}\right)_{j \in J_{k-1}}\right)\right| \leq 1$ for all $i \leq k-1$, and combining these facts we see that

$$
\left|\operatorname{Tr}_{n_{k}}\left(x_{i}\right)\right| \leq A_{k-1} \text { if } i \leq k-1 .
$$

As $q^{\delta}>6, \sum_{k=0}^{\infty} q^{-\delta k} \leq \sum_{k} 6^{-k} \leq 2$ and because

$$
\operatorname{deg} \sigma_{n_{k}} \geq q \max \left(\operatorname{deg} \sigma_{n_{k-1}}, A_{k-1}{ }^{1 /(1-\delta)}\right),
$$

it follows that

$$
\begin{aligned}
\left|\frac{1}{\operatorname{deg} \sigma_{n_{k}}}\left(\sum_{i<k} r_{i} \frac{\operatorname{Tr} \sigma_{n_{k}}\left(x_{i}\right)}{\operatorname{deg} \sigma_{n_{i}}^{\delta}}\right)\right| & \leq \frac{1}{\operatorname{deg} \sigma_{n_{k}}} \sum_{i<k} \frac{A_{k-1}}{\operatorname{deg} \sigma_{n_{i}}^{\delta}} \\
& \leq \frac{2 A_{k-1}}{\operatorname{deg} \sigma_{n_{k}}^{\delta}} \leq \frac{2}{6 \operatorname{deg} \sigma_{n_{k}}^{\delta}} .
\end{aligned}
$$

Property (i) imples that

$$
\left|r_{k} \frac{\operatorname{Tr} \sigma_{n_{k}}\left(x_{k}\right)}{\operatorname{deg} \sigma_{n_{k}}^{\delta+1}}-\frac{r_{k} \operatorname{deg} \sigma_{n_{k}}}{\operatorname{deg} \sigma_{n_{k}}^{\delta+1}}\right| \leq \frac{1}{6 \operatorname{deg} \sigma_{n_{k}}^{\delta+1}} .
$$

Since one always has $\left|\operatorname{Tr} \sigma\left(x_{i}\right)\right| \leq \operatorname{deg} \sigma$,

$$
\left|\frac{1}{\operatorname{deg} \sigma_{n_{k}}}\left(\sum_{i>k} r_{i} \frac{\operatorname{Tr} \sigma_{n_{k}}\left(x_{i}\right)}{\operatorname{deg} \sigma_{n_{i}}^{\delta}}\right)\right| \leq \sum_{i>k} \frac{1}{\operatorname{deg} \sigma_{n_{i}}^{\delta}} \leq \frac{2}{6 \operatorname{deg} \sigma_{n_{k}}^{\delta}} .
$$

Together these three estimates give the bound

$$
\sup _{k} \operatorname{deg} \sigma_{n_{k}}^{\delta}\left\|\widehat{\mu}\left(\sigma_{n_{k}}\right)-\frac{r_{k} I_{\operatorname{deg} \sigma_{n_{k}}}}{\operatorname{deg} \sigma_{n_{k}}^{\delta}}\right\|_{\infty} \leq \frac{5}{6},
$$

which certainly suffices to prove that $\left\{\sigma_{n_{k}}\right\}$ is a central $(a, 1)-I_{0}$ set.

Lemma 3.2. Suppose $G=G_{1} \times G_{2},\left\{\tau_{j}\right\} \subset \widehat{G_{1}}$ and $\left\{\sigma_{j}\right\}$ is a central $(a, 1)-I_{0}$ set in $\widehat{G_{2}}$. Then $\left\{\tau_{j} \times \sigma_{j}\right\}_{j}$ is a central $(a, 1)-I_{0}$ set in $\widehat{G}$.

Proof. If $\left(\phi(j) I_{\operatorname{deg} \tau_{j} \times \operatorname{deg} \sigma_{j}}\right)$ belongs to $l_{1-a, \infty}^{z}\left(\left\{\tau_{j} \times \sigma_{j}\right\}_{j}\right)$, then $\left(\phi(j) I_{\operatorname{deg} \sigma_{j}}\right)$ belongs to $l_{1-a, \infty}^{z}\left(\left\{\sigma_{j}\right\}_{j}\right)$. Hence there is a measure $\mu=\sum a_{k} \mu_{x_{k}}$ on $G_{2}$ whose Fourier transform interpolates $\left(\phi(j) I_{\operatorname{deg} \sigma_{j}}\right)$. But then the measure $v=\sum a_{k} \mu_{\left(e, x_{k}\right)}$ satisfies

$$
\begin{aligned}
\widehat{v}\left(\tau_{j} \times \sigma_{j}\right) & =\left(\sum a_{k} \frac{\operatorname{Tr} \tau_{j}(e) \operatorname{Tr} \sigma_{j}\left(x_{k}\right)}{\operatorname{deg} \tau_{j} \operatorname{deg} \sigma_{j}}\right) I_{\operatorname{deg} \tau_{j} \times \operatorname{deg} \sigma_{j}} \\
& =\phi(j) I_{\operatorname{deg} \tau_{j} \times \operatorname{deg} \sigma_{j}} .
\end{aligned}
$$

Thus $\left\{\tau_{j} \times \sigma_{j}\right\}_{j}$ is a central $(a, 1)-I_{0}$ set. 
Proposition 3.3. Let $G=T \times \prod_{i \in I} G_{i}$, where $T$ is a compact, abelian group and the $G_{i}$ are compact, simple, connected, simply connected Lie groups. Any infinite set of representations of $G$ of bounded degree contains an infinite, central $I_{0}$ set.

Proof. Let $\left\{\sigma_{j}\right\}$ be a sequence of distinct representations of $G$ of bounded degree. Let $\pi_{i}: G_{i} \rightarrow G$ denote the natural embedding. As $G_{i}$ contains only finitely many representations of any given degree, $\left\{\sigma_{j} \circ \pi_{i}\right\}_{j}$ is a finite set for any $i \in I$. Also, if we assume that the degrees of the representations are bounded by $2^{N}$, then the cardinality of $\left\{i: \sigma_{j} \circ \pi_{i} \neq 1\right\}$ is at most $N$ for each $j$. These facts, combined with the combinatorial argument described in detail in the proof of Theorem 2.7 of [12], show that $\left\{\sigma_{j}\right\}$ must contain an infinite subset of either of the following forms:

(i) $\left\{\tau_{j} \times \phi_{j}\right\}$, where $\tau_{j} \in \widehat{T}$ are distinct and $\phi_{j} \in \widehat{\prod_{i \in I} G_{i}}$;

(ii) $\left\{\tau_{j} \times \phi \times \chi_{j}\right\}_{j}$, where $\tau_{j} \in \widehat{T}, \phi \in \widehat{\prod_{i \in I^{\prime}} G_{i}}$ and $\left\{\chi_{j}\right\}$ are an infinite set of mutually orthogonal, non-degree one representations in $\widehat{\prod_{i \notin I^{\prime}} G_{i}}$.

If (i) holds, then by [14, Theorem 4.1] we can pick an infinite $I_{0}$ set $\left\{\tau_{j_{k}}\right\} \subset\left\{\tau_{j}\right\}$. The corresponding set $\left\{\tau_{j_{k}} \times \phi_{j_{k}}\right\}$ is central $I_{0}$ by the lemma.

In case (ii), $\left\{\chi_{j}\right\}$ is an example of an independent (or $I$ ) set in the sense of [18, Section 4], since by [5] there is an element $x_{j} \in G_{j}$ which is a zero of $\chi_{j}$. Thus $\left\{\chi_{j}\right\}$ is central $I_{0}$, and again the lemma can be invoked.

COROLlary 3.4. Let $G=T \times \prod_{i \in I} G_{i}$, where $T$ is a compact, abelian group and the $G_{i}$ are compact, simple, connected, simply connected Lie groups. Let $a<1$. Then any infinite set of representations of $G$ contains an infinite central $(a, 1)-I_{0}$ set.

Proof. Let $\left\{\sigma_{j}\right\}=\left\{\tau_{j} \times \phi_{j}\right\}$, for $\tau_{j} \in \widehat{T}$ and $\phi_{j} \in \widehat{\prod}_{i \in I} G_{i}$, be an infinite set of representations on $G$. If $\left\{\sigma_{j}\right\}$ has bounded degree, we appeal to the proposition. If it has unbounded degree, then $\left\{\phi_{j}\right\}$ is an infinite set of representations on $\prod G_{i}$ with unbounded degree. By the theorem $\left\{\phi_{j}\right\}$ contains an infinite central $(a, 1)-I_{0}$ subset, say $\left\{\phi_{j_{k}}\right\}$, and by the lemma $\left\{\tau_{j_{k}} \times \phi_{j_{k}}\right\}$ is also central $(a, 1)-I_{0}$.

Using the structure theorem for compact connected groups we can now prove the result mentioned in the introduction.

THEOREM 3.5. Let $G$ be a compact, connected group and let $a<1$. Then every infinite subset of $\widehat{G}$ contains an infinite central $(a, 1)-I_{0}$ set.

Proof. The structure theorem states that there is an epimorphism $\pi: H \equiv T \times$ $\prod_{i \in I} G_{i} \rightarrow G$, where $T$ is a compact, connected, abelian group and the $G_{i}$ are compact, simple, connected, simply connected Lie groups [19]. Let $\left\{\sigma_{j}\right\}$ be an infinite subset of $\widehat{G}$. Then $\left\{\sigma_{j} \circ \pi\right\}$ is an infinite set of representations on $H$ and consequently contains an infinite central $(a, 1)-I_{0}$ subset $\left\{\sigma_{j_{k}} \circ \pi\right\}$. It is routine to verify that $\left\{\sigma_{j_{k}}\right\}$ is central $(a, 1)-I_{0}$ as well.

REMARK 3.1. This yields a new method of proving that every infinite subset of the dual of a compact, connected group admits an infinite central $(a, 1)$-Sidon set for any $a<1$. Since a central $(1 / p, 1)$-Sidon set is also central $p$-Sidon set [15] this approach also gives a new proof of the existence of central $p$-Sidon sets for $p>1$, first established in non-abelian groups in [3].

4. Weighted $I_{0}$ sets in hypergroups. Let $K$ denote a compact, abelian hypergroup and $\widehat{K}$ its dual. We refer the reader to Jewitt's treatise [16] for basic facts about hypergroups. 
An interesting example of a compact, abelian hypergroup, which is not a group, is the space $G_{I}$ of conjugacy classes of a non-abelian compact group $G$. A function $f$ on $G$ which is constant on the conjugacy classes may be viewed as defined on the hypergroup $G_{I}$, and we will denote this function by $f^{\#}$. Jewitt showed that

$$
\widehat{G_{I}}=\left\{\frac{(\operatorname{Tr} \sigma)^{\#}}{\operatorname{deg} \sigma}: \sigma \in \widehat{G}\right\} .
$$

In [25] Sidon sets in hypergroups were investigated. Weighted Sidon sets were introduced in [13], and this definition can also be naturally extended to $I_{0}$ sets. Again we need to introduce weighted $l_{\infty}$ spaces: Given $a \in \mathbb{R}$ and $E \subseteq \widehat{K}$ let

$$
l_{a, \infty}(E)=\left\{\left(a_{\chi}\right)_{\chi \in E}:\left\|\left(a_{\chi}\right)\right\|_{a, \infty} \equiv \sup _{\chi \in E}\left\{\left|a_{\chi}\right|\|\chi\|_{2}^{-2 a}\right\}<\infty\right\} .
$$

The exponent $2 a$ is a notational convenience. Of course, if $a=0$, then this is the usual space $l_{\infty}(E)$. As the characters of $K$ are always bounded from above, if $\inf \left\{\|\chi\|_{2}: \chi \in\right.$ $E\}>0$ (as is the case when $K$ is a group, for example), the spaces $l_{a, \infty}$ are identical. But for many hypergroups, $l_{a, \infty}(E)$ is a proper subset of $l_{b, \infty}(E)$ for $a>b$.

Definition 4.1. Let $K$ be a compact, abelian hypergroup. A subset $E \subseteq \widehat{K}$ is called an $(a, 1)$-Sidon (resp. $\left.(a, 1)-I_{0}\right)$ set if whenever $\phi \in l_{1-a, \infty}(E)$, there is a (resp. discrete) measure $\mu$ on $K$ such that $\widehat{\mu}(\chi)=\phi(\chi)$ for all $\chi \in E$.

REMARK 4.1. Suppose $\delta_{C(x)}$ denotes the point mass measure on $G_{I}$ at the point which is the conjuacy class containing $x \in G$ and $\mu=\sum b_{k} \delta_{C\left(x_{k}\right)}$. Let $v \in M(G)$ be given by $v=\sum b_{k} \mu_{x_{k}}$. If $\sigma \in \widehat{G}$ and $\chi=(\operatorname{Tr} \sigma)^{\#} / \operatorname{deg} \sigma$ is the corresponding character on $G_{I}$, then $\widehat{\mu}(\chi) I_{\operatorname{deg} \sigma}=\widehat{v}(\sigma)$. Thus $E \subseteq \widehat{G}$ is central $(2 a-1,1)-I_{0}$ if and only if $E^{\#} \subseteq \widehat{G_{I}}$ is $(a, 1)-I_{0}$ in the hypergroup sense. This is further motivation for taking the interpolating measures to be sums of orbital measures in the definition of central weighted $I_{0}$ sets for non-abelian groups.

Weighted $I_{0}$ sets on hypergroups can be characterised in a manner analogous to Proposition 2.3.

Proposition 4.1. Let $K$ be a compact abelian hypergroup. The following are equivalent for $E \subset \widehat{K}$ :

(1) The set $E$ is an $(1-a, 1)-I_{0}$ set.

(2) There is a constant $C$ such that whenever $\phi \in l_{a, \infty}(E)$ there is a discrete measure $\mu$ such that $\widehat{\mu}(\chi)=\phi(\chi)$ for all $\chi \in E$ and $\|\mu\| \leq C\|\phi\|_{a, \infty}$.

(3) For every $0<\varepsilon<1$ (equivalently, there exists $0<\varepsilon<1$ ) for which there is a constant $C$ so that whenever $\phi \in l_{a, \infty}(E)$ there is a discrete measure $\mu$ such that $\left\|\left.\widehat{\mu}\right|_{E}-\phi\right\|_{a, \infty} \leq \varepsilon\|\phi\|_{a, \infty}$ and $\|\mu\| \leq C\|\phi\|_{a, \infty}$.

(4) For every $0<\varepsilon<1$ (equivalently, there exists $0<\varepsilon<1$ ) for which there is a constant $C$ so that for each choice of $\left\{r_{\chi}\right\}_{\chi \in E}, r_{\chi}= \pm 1$, there is a discrete measure $\mu$ such that $\|\mu\| \leq C$ and

$$
\left\|\left(\widehat{\mu}(\chi)-r_{\chi}\|\chi\|_{2}^{2 a}\right)_{\chi \in E}\right\|_{a, \infty} \leq \varepsilon
$$

Proposition 4.2. Any finite set $E$ in $\widehat{K}$ is $I_{0}$. 
Proof. This is an easy consequence of the compactness of $K$ and the fact that finite sets are Sidon. Given a finite subset $E$ of $\widehat{K}$ and $\phi \in l_{\infty}(E)$ of norm at most one, choose a measure $\mu$ such that $\widehat{\mu}(\chi)=\phi(\chi)$ for all $\chi \in E$. For each $x \in K$ obtain a neighbourhood $U_{x}$ such that

$$
|\chi(x)-\chi(y)|<\frac{\varepsilon}{\|\mu\|} \text { for all } y \in U_{x} \text { and } \chi \in E .
$$

Choose a finite subcover $U_{x_{1}}, \ldots, U_{x_{n}}$. Without loss of generality we can assume these sets are disjoint. Set $v=\sum_{j=1}^{n} \mu\left(U_{x_{j}}\right) \delta_{x_{j}}$. It is a routine exercise to verify that $|\widehat{v}(\chi)-\phi(\chi)|<\varepsilon$ for all $\chi \in E$.

It is known that every infinite subset of $\widehat{K}$ contains an infinite set that is $(a, 1)$-Sidon for all $a<1$ [13]. In this section we will prove a partial extension of this.

DEFINITION 4.2. We will say that the compact, abelian hypergroup $K$ has the pointwise boundedness property if every neighbourhood of $e$ contains infinitely many points $x \in K$ having the property that there is a constant $C(x)$ satisfying $|\chi(x)| \leq$ $C(x)\|\chi\|_{2}$ for all $\chi \in \widehat{K}$.

First, we give some examples of hypergroups which have this property.

EXAMPLE 4.1. The hypergroup of conjugacy classes of a compact, semi-simple, connected, simply connected Lie group has the pointwise boundedness property.

Proof. This is a consequence of the Weyl character formula (see [24]) from which one can conclude that

$$
\left|\frac{\operatorname{Tr} \sigma(x)}{\operatorname{deg} \sigma}\right| \leq \frac{C(x)}{\operatorname{deg} \sigma}
$$

for the dense set of regular elements in the group. When $\chi=\operatorname{Tr} \sigma / \operatorname{deg} \sigma,\|\chi\|_{2}=$ $(\operatorname{deg} \sigma)^{-1}$; hence the result is clear.

EXAMPLE 4.2. The hypergroup whose dual is a set of (normalised) Jacobi polynomials, $P_{n}^{\alpha, \beta} / P_{n}^{\alpha, \beta}(1)$, has the pointwise boundedness property.

Proof. This can be seen from the asymptotic behaviour of the Jacobi polynomials. It is known [23, p. 167] that

$$
P_{n}^{\alpha, \beta}(\cos \theta)= \begin{cases}\theta^{-\alpha-1 / 2} O\left(n^{-1 / 2}\right) & \text { if } n^{-1} \leq \theta \leq \pi / 2 \\ O\left(n^{\alpha}\right) & \text { if } 0 \leq \theta \leq n^{-1}\end{cases}
$$

As a result, $\left\|P_{n}^{\alpha, \beta}\right\|_{2} \geq O\left(n^{-1 / 2}\right)$. Provided $x \neq \pm 1$, the inequalities above imply that there is a constant $C(x)$ such that $\left|P_{n}^{\alpha, \beta}(x)\right| \leq C(x)\left\|P_{n}^{\alpha, \beta}\right\|_{2}$ for all $n$ sufficiently large.

THEOREM 4.3. Let $K$ be a compact, abelian hypergroup having the pointwise boundedness property and let $a<1$. Suppose $\left\{\chi_{n}\right\}_{n=1}^{\infty}$ is a subset of $\widehat{K}$ satisfying $\inf _{n}\left\|\chi_{n}\right\|_{2}=0$. Then $\left\{\chi_{n}\right\}$ contains an infinite central $(a, 1)-I_{0}$ set.

Proof. Put $\delta=1-a<1 / 2$. Choose $n_{1}$ such that $\left\|\chi_{n_{1}}\right\|_{2} \leq 1 / 4$. As $\chi_{n_{1}}$ is continuous and $K$ has the pointwise boundedness property we may select $x_{1} \in K$ and a constant $A_{1}$ 
such that $\left|\chi_{n_{1}}\left(x_{1}\right)-1\right|<1 / 4$ and $\left|\chi\left(x_{1}\right)\right| \leq A_{1}\|\chi\|_{2}$ for all $\chi \in \widehat{K}$. Because $2 \delta-1<0$, we may pick $n_{2}>n_{1}$ so that

$$
32 A_{1}\left\|\chi_{n_{2}}\right\|_{2}^{2 \delta} \leq 4 A_{1}\left\|\chi_{n_{1}}\right\|_{2}^{2 \delta} \leq\left\|\chi_{n_{2}}\right\|_{2}^{2 \delta-1} .
$$

Then choose $x_{2} \neq x_{1}$ and $A_{2}$ such that $\left|\chi_{n_{2}}\left(x_{2}\right)-1\right|<1 / 4$ and $\left|\chi\left(x_{2}\right)\right| \leq A_{2}\|\chi\|_{2}$ for all $\chi \in \widehat{K}$.

Repeating this procedure inductively constructs an infinite sequence of integers $n_{1}<n_{2}<\cdots$, distinct points $x_{1}, x_{2}, \cdots \in K$ and constants $A_{1}, A_{2}, \ldots$, satisfying the following:

(i) $\left|\chi_{n_{j}}\left(x_{j}\right)-1\right|<1 / 4,\left|\chi\left(x_{j}\right)\right| \leq A_{j}\|\chi\|_{2}$ for all $\chi \in \widehat{K}, j \geq 1$;

(ii) $\sum_{k<j} A_{k}\left\|\chi_{n_{k}}\right\|_{2}^{2 \delta} \leq\left\|\chi_{n_{j}}\right\|_{2}^{2 \delta-1} / 4$ for $j \geq 2$; and

(iii) $\left\|\chi_{n_{j}}\right\|_{2}^{2 \delta} \leq\left\|\chi_{n_{j-1}}\right\|_{2}^{2 \delta} / 8$ for $j \geq 2$.

We will now prove that $\left\{\chi_{n_{k}}\right\}$ is an $(a, 1)-I_{0}$ set by verifying property (4) of Proposition 4.1. So let $\left\{r_{k}\right\}$ be a choice of signs and consider the discrete measure

$$
\mu=\sum_{k=1}^{\infty} r_{k}\left\|\chi_{n_{k}}\right\|_{2}^{2 \delta} \delta_{x_{k}}
$$

This is a finite measure since

$$
\sum_{k}\left\|\chi_{n_{k}}\right\|_{2}^{2 \delta} \leq \sum_{k} 8^{-k+1}\left\|\chi_{n_{1}}\right\|_{2}^{2 \delta}<\infty
$$

Since $\widehat{\mu}\left(\chi_{n_{j}}\right)=\sum_{k} r_{k}\left\|\chi_{n_{k}}\right\|_{2}^{2 \delta} \chi_{j}\left(x_{k}\right)$ we have

$$
\begin{aligned}
\left|\widehat{\mu}\left(\chi_{n_{j}}\right)-r_{j}\left\|\chi_{n_{j}}\right\|_{2}^{2 \delta}\right| & =\left|\sum_{k \neq j} r_{k}\left\|\chi_{n_{k}}\right\|_{2}^{2 \delta} \chi_{n_{j}}\left(x_{k}\right)\right|+\left|r_{j}\left\|\chi_{n_{j}}\right\|_{2}^{2 \delta}\left(\chi_{n_{j}}\left(x_{j}\right)-1\right)\right| \\
& \leq\left|\sum_{k \neq j} r_{k}\left\|\chi_{n_{k}}\right\|_{2}^{2 \delta} \chi_{n_{j}}\left(x_{k}\right)\right|+\left\|\chi_{n_{j}}\right\|_{2}^{2 \delta} / 4 .
\end{aligned}
$$
obtain

To bound the sum over $k<j$ we use the fact that $\left|\chi_{n_{j}}\left(x_{k}\right)\right| \leq A_{k}\left\|\chi_{n_{j}}\right\|_{2}$ and (ii) to

$$
\left|\sum_{k<j} r_{k}\left\|\chi_{n_{k}}\right\|_{2}^{2 \delta} \chi_{n_{j}}\left(x_{k}\right)\right| \leq \sum_{k<j} A_{k}\left\|\chi_{n_{k}}\right\|_{2}^{2 \delta}\left\|\chi_{n_{j}}\right\|_{2} \leq \frac{\left\|\chi_{n_{j}}\right\|_{2}^{2 \delta}}{4} .
$$

For the sum over $k>j$ we note that $\left|\chi_{n_{j}}\left(x_{k}\right)\right| \leq 1$; thus

$$
\left|\sum_{k>j} r_{k}\left\|\chi_{n_{k}}\right\|_{2}^{2 \delta} \chi_{n_{j}}\left(x_{k}\right)\right| \leq \sum_{k=j+1}^{\infty}\left\|\chi_{n_{k}}\right\|_{2}^{2 \delta} \leq\left\|\chi_{n_{j}}\right\|_{2}^{2 \delta} \sum_{k=1}^{\infty} 8^{-k} \leq \frac{\left\|\chi_{n_{j}}\right\|_{2}^{2 \delta}}{4} .
$$

Hence the choice of $\left\{x_{j}\right\}$ ensures that

$$
\left|\widehat{\mu}\left(\chi_{n_{j}}\right)-r_{j}\left\|\chi_{n_{j}}\right\|_{2}^{2 \delta}\right| \leq \frac{3}{4}\left\|\chi_{n_{j}}\right\|_{2}^{2 \delta},
$$

and this establishes property (4). 
REMARK 4.2. Note that the proof actually shows that the pointwise boundedness property is not needed for all characters in $\widehat{K}$ but only those from the infinite set $\left\{\chi_{n}\right\}$.

COROLlary 4.4. Suppose $K$ is an infinite hypergroup whose dual is a set of Jacobi polynomials, $P_{n}^{\alpha, \beta}(x) / P_{n}^{\alpha, \beta}(1)$ with $\alpha>-1 / 2$. If $a<1$, then any infinite set of characters contains an infinite $(a, 1)-I_{0}$ set.

Proof. We have already observed that such a hypergroup has the pointwise boundedness property. Since $P_{n}^{\alpha, \beta}(1)=O\left(n^{\alpha}\right)$, if $\alpha>-1 / 2$ then any set of characters $\left\{\chi_{n}\right\}$ satisfies $\inf _{n}\left\|\chi_{n}\right\|_{2}=0$.

ACKNOWLEDGEMENT. This research was partially supported by NSERC.

\section{REFERENCES}

1. D. Cartwright and J. McMullen, A structural criterion for the existence of infinite Sidon sets, Pac. J. Math. 96 (1981), 301-317.

2. W. Connett and A. Schwartz, Subsets of $\mathbb{R}$ which support hypergroups with polynomial characters, J. Comput. Appl. Math. 65 (1995), 73-84.

3. T. Dooley, Central lacunary sets for Lie groups, J. Aust. Math. Soc. 45 (1988), 30-45.

4. J. Galindo and S. Hernandez, The concept of boundedness and the Bohr compactification of a MAP abelian group, Fund. Math. 159 (1999), 195-218.

5. P. Gallagher, Zeroes of group characters, Math. Z. 87 (1965), 363-364.

6. B. Givens and K. Kunen, Chromatic numbers and Bohr topologies, Topol. Appl. 131 (2003), 189-202.

7. C. Graham and K. Hare, $\varepsilon$-Kronecker and $I_{0}$ sets in abelian groups, IV: Interpolation by non-negative measures, Studia Math. 177 (2006), 9-24.

8. C. Graham and K. Hare, $I_{0}$ sets for compact, connected groups: Interpolaton with measures that are non-negative or of small support, J. Aust. Math. Soc. 84 (2008), 199-215.

9. C. Graham, K. Hare and T. Korner, $\varepsilon$-Kronecker and $I_{0}$ sets in abelian groups, II: Sparseness of products of $\varepsilon$-Kronecker sets, Math. Proc. Camb. Phil. Soc. 140 (2006), 491-508.

10. C. Graham and A. Lau, Relative weak compactness of orbits in Banach spaces associated with locally compact groups, Trans. Am. Math. Soc. 359 (2007), 1129-1160.

11. D. Grow and K. Hare, The independence of characters on non-abelian groups, Proc. Am. Math. Soc. 132 (2004), 3641-3651.

12. K. Hare, Central Sidonicity for compact Lie groups, Ann. Inst. Fourier (Grenoble) $\mathbf{4 5}$ (1995), 547-564.

13. K. Hare, Sidonicity in compact, abelian hypergroups, Colloq. Math. 76 (1998), 171-180.

14. K. Hare and T. Ramsey, $I_{0}$ sets in non-abelian groups, Math. Proc. Camb. Phil. Soc. 135 (2003), 81-98.

15. K. Hare and D. Wilson, Weighted p-Sidon sets, J. Aust. Math. Soc. 61 (1996), 73-95.

16. R. Jewitt, Spaces with an abstract convolution of measures, Adv. Math. 18 (1975), 1-101.

17. K. Kunen and W. Rudin, Lacunarity and the Bohr topology, Math. Proc. Camb. Phil. Soc. 126 (1999), 117-137.

18. W. Parker, Central Sidon and central $\Lambda_{p}$ sets, J. Aust. Math. Soc. 14 (1972), 62-74.

19. J. Price, Lie groups and compact groups, London Mathematical Society Lecture Notes Series 25 (Cambridge University Press, Cambridge, UK, 1977).

20. D. Ragozin, Central measures on compact simple Lie groups, J. Func. Anal. 10 (1972), $212-229$.

21. T. Ramsey, Comparisons of Sidon and $I_{0}$ sets, Colloq. Math. 70 (1996), 103-132.

22. D. Rider, Central lacunary sets, Monatsh. Math. 76 (1972), 328-338.

23. G. Szego, Orthogonal polynomials (American Mathematical Society, New York, 1975).

24. V. Varadarajan, Lie groups, Lie algebras and their representations (Springer, New York, 1984).

25. R. Vrem, Independent sets and lacunarity for hypergroups, J. Aust. Math. Soc. 50 (1991) $171-188$. 